\title{
Parametric Excitation of an Optically Silent Goldstone-Like Phonon Mode
}

\author{
Dominik M. Juraschek $\odot,{ }^{1, *}$ Quintin N. Meier $\odot,{ }^{2}$ and Prineha Narang $\oplus^{1, \dagger}$ \\ ${ }^{1}$ Harvard John A. Paulson School of Engineering and Applied Sciences, Harvard University, Cambridge, Massachusetts 02138, USA \\ ${ }^{2}$ Department of Materials, ETH Zurich, CH-8093 Zürich, Switzerland
}

(Received 16 November 2019; revised manuscript received 24 January 2020; accepted 19 February 2020; published 16 March 2020)

\begin{abstract}
It has recently been indicated that the hexagonal manganites exhibit Higgs- and Goldstone-like phonon modes that modulate the amplitude and phase of their primary order parameter. Here, we describe a mechanism by which a silent Goldstone-like phonon mode can be coherently excited, which is based on nonlinear coupling to an infrared-active Higgs-like phonon mode. Using a combination of first-principles calculations and phenomenological modeling, we describe the coupled Higgs-Goldstone dynamics in response to the excitation with a terahertz pulse. Besides theoretically demonstrating coherent control of crystallographic Higgs and Goldstone excitations, we show that the previously inaccessible silent phonon modes can be excited coherently with this mechanism.
\end{abstract}

DOI: 10.1103/PhysRevLett.124.117401

Order parameters are physical observables that are used to quantify the different states of matter. Their amplitudes and phases can be excited by external stimuli, such as a laser pulse, leading to exotic states of matter that cannot be accessed in equilibrium [1]. Two particular excitations are Higgs and Goldstone modes, which correspond to the modulation of the amplitude and phase of an order parameter that breaks a continuous symmetry. Originally discussed in the context of particle physics [2] and superconductivity [3], Higgs and Goldstone excitations were found in cold atom systems, such as superfluids [4-8] or supersolids [9]. Higgs and Goldstone modes are well studied in superconductors today [10-21], and similar manifestations have recently been reported in charge density wave systems [22-24], antiferromagnets [25-27], and excitonic insulators [28]. It has been suggested that the optical and acoustic vibrational modes of solids can be considered Higgs and Goldstone excitations of the crystal lattice [29]. Several complex transition metal oxide compounds have shown signatures of optical Goldstone-like phonon modes that live in their symmetry-broken potential energy landscape [30-35].

Coherent control over Raman-active phonons via impulsive stimulated Raman scattering using visible light pulses is well established [36,37]. Recently, the excitation of infrared-active phonons with large amplitudes via IR absorption has become feasible through the development of

Published by the American Physical Society under the terms of the Creative Commons Attribution 4.0 International license. Further distribution of this work must maintain attribution to the author(s) and the published article's title, journal citation, and DOI. high intensity terahertz and mid-IR sources. This progress has enabled selective control over the dynamics of the crystal lattice through nonlinear phonon interactions using ionic Raman scattering [38-40] and two-particle absorption mechanisms [41-46]. In contrast, phonon modes that do not respond to IR absorption or Raman scattering techniques, so-called silent modes, can only be detected through hyper-Raman scattering. As hyper-Raman scattering is a third-order interaction of the electric field component of light with a phonon mode, it is inefficient, and no coherent excitation has been achieved yet.

Higgs and Goldstone modes, similar to silent phonon modes, are challenging to excite and coherently address, because they often do not directly couple to light. In this work, we overcome this hurdle by synthesizing advances from two parallel fields, order-parameter physics of continuous symmetry-broken systems and coherent control of crystal lattice dynamics. Specifically, we demonstrate that the low-frequency silent Goldstone-like optical phonon mode in indium manganite $\left(\mathrm{InMnO}_{3}\right)$ can be coherently excited by coupling nonlinearly to the low-frequency Higgs-like optical phonon mode. In $\mathrm{InMnO}_{3}$, this Higgslike phonon mode is IR active and can be driven resonantly with a terahertz pulse. We depict the two phonon modes in Fig. 1. The underlying mechanism of the excitation of the Goldstone-like phonon mode is a parametric downconversion process from an optical phonon mode to another optical phonon mode at the Brillouin zone center, as shown in Fig. 2(a), enabled through the nonlinear phonon coupling. This process results in a coherent excitation of the silent Goldstone-like phonon mode with large amplitude. The mechanism of parametric down-conversion has previously been limited to the conventional decay of gapped (or optical) excitations into gapless (or acoustic) excitations 


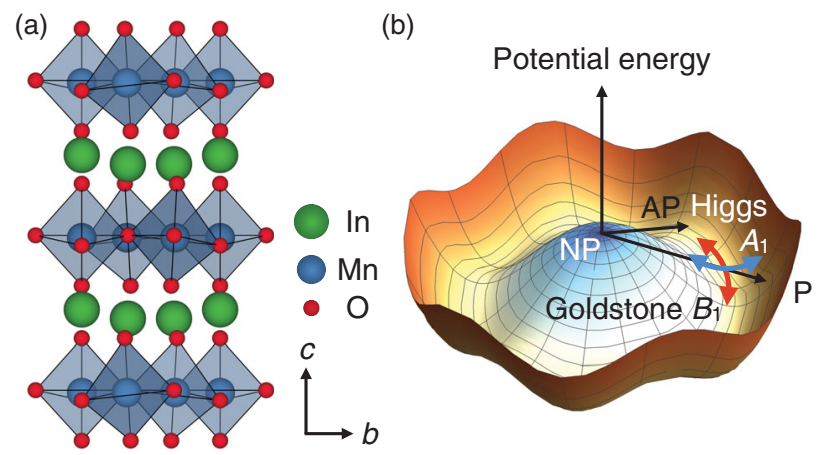

FIG. 1. Crystallographic properties of $\mathrm{InMnO}_{3}$. (a) View of the $b c$ plane of the crystal in its hexagonal phase with space group $P 6_{3} \mathrm{~cm}$. (b) Mexican hat potential energy landscape of the lattice with nonpolar (NP), antipolar (AP), and polar (P) phases indicated. Blue and red arrows depict the Higgs-like $A_{1}$ and Goldstone-like $B_{1}$ modes in the polar phase.

with high wave vectors, as shown in Fig. 2(b), where experimental demonstrations include phonons in bismuth [47], magnons in calcium ruthenate [26], and charge density waves in blue bronze [22].

Structural properties of $\mathrm{InMnO}_{3}$. - $\mathrm{InMnO}_{3}$ crystallizes in the hexagonal manganite structure shown in Fig. 1(a): The potential energy landscape of the lattice displacement forms a buckled Mexican hat that hosts a nonpolar phase at its center, and polar and antipolar phases at the minima and maxima of its brim [48-50]; see Fig. 1(b). The primary order parameter corresponds to a tilting of the manganese oxygen bipyramids and a simultaneous buckling of the indium atoms. This two-dimensional order parameter couples to a ferroelectric displacement with polarization along the $c$ axis of the crystal. This coupling is responsible for the minima in the brim of the Mexican hat and stabilizes the improper ferroelectric ground state [49]. $\mathrm{InMnO}_{3}$ is a special member of the hexagonal manganite family and has a particularly flat brim which can be tuned using chemical
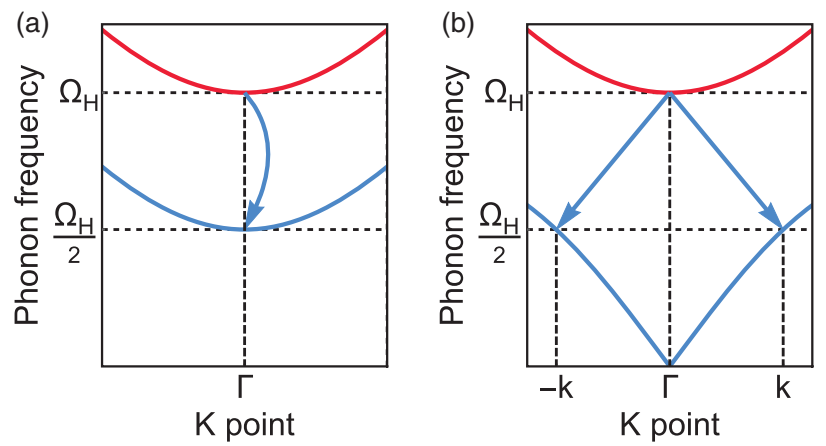

FIG. 2. Schematic parametric down-conversion mechanisms of the resonantly driven Higgs mode (red). (a) Down-conversion to the Goldstone mode (blue) at the Brillouin zone center, as proposed in this work. (b) Conventional parametric decay into acoustic modes (blue) with opposite wave vectors throughout the Brillouin zone. doping or strain [51,52]. The 30 atom primitive unit cell of the polar phase with $P 6_{3} \mathrm{~cm}$ space group $(6 \mathrm{~mm}$ point group) hosts 90 phonon modes with irreducible representations $A_{1,2}, B_{1,2}$, and $E_{1,2}$. It has recently been indicated that the low-frequency $A_{1}$ mode at $4 \mathrm{THz}$ and $B_{1}$ mode at $2.4 \mathrm{THz}$ correspond to the amplitude and phase modulations of the primary order parameter and can therefore be considered to have Higgs and Goldstone character [34]. The Higgs-like $A_{1}$ mode is Raman active and IR active along the hexagonal crystal axis, while the Goldstone-like $B_{1}$ mode is silent (neither Raman nor IR active). For simplicity, we will in the following refer to the $A_{1}$ mode as "Higgs" mode and to the $B_{1}$ mode as "Goldstone" mode.

Density functional theory calculations.-We begin by calculating the structural properties of $\mathrm{InMnO}_{3}$ from first principles using the density functional theory formalism as implemented in the ABINIT code [53]. For the exchangecorrelation functional, we apply the local density approximation plus Hubbard $U(\mathrm{LDA}+U)$ [54] and we use the default projector augmented wave (PAW) method [55] with GPAW pseudopotentials [56]. Good convergence was achieved using a cutoff energy of $30 \mathrm{Ha}$ and a gammacentered $k$-point mesh of $6 \times 6 \times 4$. We apply a Hubbard $U$ of $4.5 \mathrm{eV}$ and exchange $J$ of $0.45 \mathrm{eV}$ using frustrated antiferromagnetic ordering [57]. We obtain the phonon eigenfrequencies, eigenvectors, and Born effective charge tensors using density functional perturbation theory $[58,59]$. In order to obtain the nonlinear phonon couplings, we calculate the total energy as a function of ion displacements along the normal mode coordinates of the Higgs and Goldstone modes and then fit the resulting two-dimensional energy landscape to the potential $V$ in Eq. (2).

Nonlinear Higgs-Goldstone dynamics.-To investigate the time evolution of the Higgs and Goldstone modes in response to a pulsed terahertz excitation, we numerically solve their equations of motion,

$$
\ddot{Q}_{i}+\kappa_{i} \dot{Q}_{i}+\frac{\partial V}{\partial Q_{i}}-Z_{i} E=0
$$

where $Q_{i}$ is the normal mode coordinate (or amplitude) of the Higgs $(i=H)$ and Goldstone $(i=G)$ mode in units of $\AA \sqrt{\mathrm{amu}}$, where amu is the atomic mass unit. $\kappa_{i}$ is the phonon linewidth, $Z_{i}$ is the mode effective charge, $V(Q)$ is the anharmonic phonon potential, and $E$ is the electric field component of the pulse. The mode effective charge is given by $Z_{i}=\sum_{n} Z_{n}^{*} \mathbf{q}_{i, n} / \sqrt{M_{n}}$, where $Z_{n}^{*}$ is the Born effective charge tensor of atom $n$ in units of the elementary charge $e$, $\mathbf{q}_{i, n}$ is its eigenvector, and $M_{n}$ its atomic mass [58]. $Z_{i}$ is only nonzero if $i$ is an IR-active phonon mode. The displacement of each individual ion $\mathbf{d}_{i, n}$ along the phonon modes can be calculated as $\mathbf{d}_{i, n}=\mathbf{q}_{i, n} Q_{i}(t) / \sqrt{M_{n}}$.

As $A_{1} \subset\left[B_{1} \times B_{1}\right]$, the lowest-order symmetry-allowed nonlinear phonon coupling between the Higgs and Goldstone modes is of the form $Q_{H} Q_{G}^{2}$. A phonon coupling 
squared in the IR-active mode and linear in the other one that is required for ionic Raman scattering [39,42] is forbidden by symmetry for the silent $B_{1}$ mode here. The potential energy of the phonons can therefore be written in a minimal model as

$$
V_{\min }(Q)=\frac{\Omega_{H}^{2}}{2} Q_{H}^{2}+\frac{\Omega_{G}^{2}}{2} Q_{G}^{2}+c Q_{H} Q_{G}^{2},
$$

where $\Omega_{H}$ and $\Omega_{G}$ are the phonon eigenfrequencies given in $2 \pi \times \mathrm{THz}$, and $c$ is their linear-quadratic coupling coefficient given in $\mathrm{meV} /(\AA \sqrt{\mathrm{amu}})^{3}$. For accuracy we include all anharmonicities and nonlinear phonon couplings up to fourth order in the phonon amplitude, which can be written as $\tilde{V}=d Q_{H}^{3}+e Q_{H}^{2} Q_{G}^{2}+f Q_{H}^{4}+g Q_{G}^{4}$. We find the coupling coefficients $d$ to $g$ to be small compared to $c$. Substituting $V=V_{\min }+\tilde{V}$ into Eq. (1), we obtain

$$
\begin{gathered}
\ddot{Q}_{H}+\kappa_{H} \dot{Q}_{\mathrm{IR}}+\Omega_{H}^{2} Q_{H}+c Q_{G}^{2}+\frac{\partial \tilde{V}}{\partial Q_{H}}-Z_{H} E=0, \\
\ddot{Q}_{G}+\kappa_{G} \dot{Q}_{G}+\left(\Omega_{G}^{2}+2 c Q_{H}\right) Q_{G}+\frac{\partial \tilde{V}}{\partial Q_{G}}=0 .
\end{gathered}
$$

The nonlinear phonon coupling acts as a temporal modulation of the eigenfrequency of the Goldstone mode in Eq. (4), $\tilde{\Omega}_{G}^{2}(t)=\Omega_{G}^{2}+2 c Q_{H}(t)$. When $Q_{H}$ oscillates at double the frequency of $\Omega_{G}, Q_{G}$ is parametrically amplified. Once the Goldstone mode is excited, $Q_{G}^{2}$ reciprocally acts as a driving force for the Higgs mode in Eq. (3).

Note that the phonon modes are detuned from the resonance condition $\Omega_{G}=\Omega_{H} / 2$ by $0.4 \mathrm{THz}$. As we will see, this mismatch is compensated by the broadening of the phonon frequencies due to their natural linewidths and due to the temporal envelope that the IR-active Higgs mode obtains through the resonant driving by the terahertz pulse. In addition to the parametric down-conversion described here, coupling of the Higgs mode to higher-frequency $A_{1}$ modes of the form $Q_{H}^{2} Q_{A_{1}}$ may lead to a phonon upconversion according to sum-frequency ionic Raman scattering [42]. This process is possible for coupling to the 7 and $9 \mathrm{THz} A_{1}$ modes in $\mathrm{InMnO}_{3}$. As for other higher-order anharmonicities, this process would lead to quantitative changes in the amplitude of the Higgs mode, but not qualitatively influence the coupled Higgs-Goldstone dynamics described here. This coupling to other modes is implicitly taken into account in the phonon linewidths.

We model the electric field component of the terahertz pulse as $E(t)=E_{0} \exp \left\{-t^{2} /\left[2(\tau / \sqrt{8 \ln 2})^{2}\right]\right\} \cos \left(\omega_{0} t\right)$, where $E_{0}$ is the peak electric field, $\omega_{0}$ is the center frequency, and $\tau$ is the full width at half maximum duration of the pulse [42]. In order to couple to the dipole moment of the Higgs mode, the electric field component has to be aligned with the hexagonal axis of the crystal. We choose experimentally feasible values for $E_{0}$ and $\tau$ for this
TABLE I. Calculated eigenfrequencies $\Omega_{H / G}$, coupling coefficients $c$ to $g$, mode effective charge $Z_{H}$, peak electric field $E_{0}$, and full width at half maximum pulse duration $\tau$ of the laser

\begin{tabular}{|c|c|c|c|c|c|c|c|c|c|}
\hline$\Omega_{H} /(2 \pi)$ & $\Omega_{G} /(2 \pi)$ & $c$ & $d$ & $e$ & $f$ & $g$ & $Z_{H}$ & $E_{0}$ & $\tau$ \\
\hline $4.0 \mathrm{THz}$ & $2.4 \mathrm{THz}$ & 9.2 & 20.9 & 1.5 & 0.2 & 0.5 & 2.7 & $\mathrm{MVcm}$ & $0.5 \mathrm{ps}$ \\
\hline
\end{tabular}
pulse. The coupling coefficients are in units of $\mathrm{meV} /(\AA \sqrt{\mathrm{amu}})^{n}$, where $n$ is the order of phonon amplitude.

frequency range [60]. We list the used parameters in Table I. As the calculation of phonon linewidths is computationally challenging for larger unit cells (calculations for 2 or 4 atom unit cells have been performed [61], compared to the 30 atom unit cell in the present case), and no experimental data on phonon linewidths in $\mathrm{InMnO}_{3}$ exists to our knowledge, we take $\kappa_{H}$ and $\kappa_{G}$ as free parameters of the model within possible experimental boundaries. Raman and IR studies on closely related hexagonal yttrium manganite $\left(\mathrm{YMnO}_{3}\right)$ show that phonon linewidths lie within 3\%-20\% of the phonon frequency, $\kappa_{i} \approx 0.03$ to $0.2 \times \Omega_{i} /(2 \pi)$ [62]. Finally, we add a thermal noise $\left(2 k_{B} T \kappa_{G / H}^{2}\right)^{1 / 2} \xi(t)$ to the equations to ensure that the initial amplitudes of the Higgs and Goldstone modes are nonzero at room temperature. Here, $\xi$ is a Gaussian random variable with variance one, $k_{B}$ is the Boltzmann constant, and $T$ is the temperature $[63,64]$.

We show representative dynamics of the Higgs and Goldstone modes after the optical excitation according to Eqs. (3) and (4) in Figs. 3(a) and 3(b), where we have chosen linewidths of $10 \%$ of the phonon frequencies. While the amplitude of the Higgs mode $Q_{H}$ reaches its maximum during the duration of the terahertz pulse in Fig. 3(a), the amplitude of the Goldstone mode $Q_{G}$ only starts building up with a time delay of 2 ps due to the parametric amplification by the Higgs mode. The delayed buildup is also visible in the time evolution of the vibrational energy contained in each mode in Fig. 3(b). The vibrational energy contained in the resonantly driven Higgs mode (scaling quadratically in both the eigenfrequency and amplitude) is roughly 3 times larger than that contained in the Goldstone mode. Note that the time delay of the buildup of the Goldstone mode amplitude depends on various factors, such as the relative frequencies of the phonon modes, their dampings, and the strength of their nonlinear coupling.

As the parametric excitation requires an initial nonzero value of the Goldstone mode, the outcome of Eqs. (3) and (4) depends stochastically on the thermal noise background. In order to estimate the magnitude of induced atomic motion, we therefore sample the phonon amplitudes $Q_{H}$ and $Q_{G}$ for a large number of linewidths $\kappa_{H}$ and $\kappa_{G}$ between $3 \%$ and $20 \%$ of the respective eigenfrequencies. The low-frequency Higgs and Goldstone modes involve mainly motions of the indium and oxygen ions. We extract the root mean squares of the largest ionic displacements, 
(a)

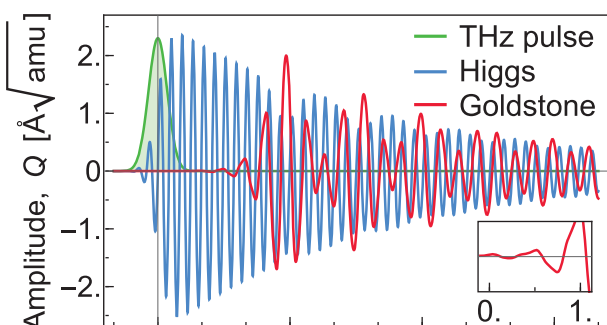

(b)

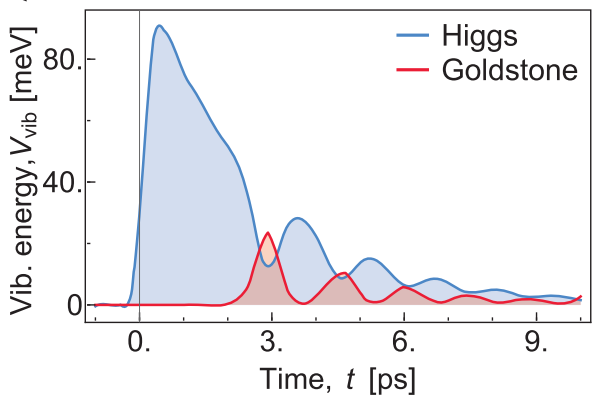

(c)

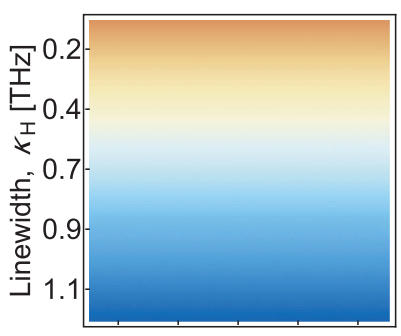

(d)

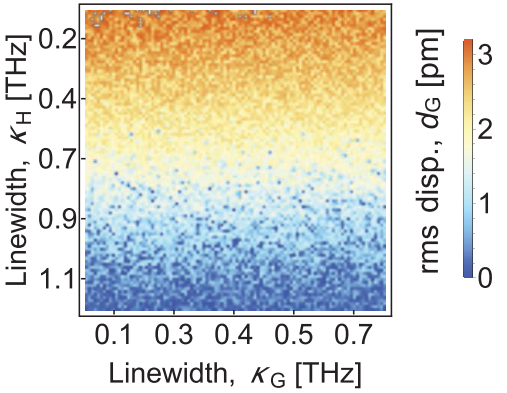

FIG. 3. Phonon dynamics in response to the excitation by a pulse with center frequency $\omega_{0}=4 \mathrm{THz}$, duration $\tau=0.5 \mathrm{ps}$, and peak field $E_{0}=1 \mathrm{MV} / \mathrm{cm}$. (a) Time evolution of the amplitudes $Q$ of the $4 \mathrm{THz}$ Higgs and $2.4 \mathrm{THz}$ Goldstone modes for linewidths of $\kappa_{H}=0.4 \mathrm{THz}$ and $\kappa_{G}=0.24 \mathrm{THz}$. We show the carrier envelope of the pulse schematically. The inset shows the Goldstone mode resolved in the region between 0 and 1 ps. (b) Evolution of the vibrational energy per unit cell $V_{\text {vib }}$ contained in each of the phonon modes in units of $\mathrm{eV}$, extracted from the root mean squares (rms's) of the phonon amplitudes. (c),(d) rms's of the largest ionic displacements along the Higgs $\left(d_{H}\right)$ and Goldstone $\left(d_{G}\right)$ modes for different $\kappa_{H}$ and $\kappa_{G}$ ranging between $3 \%$ and $20 \%$ of their phonon frequencies.

$d_{H / G}=\max _{n}\left|\mathbf{d}_{H / G, n} / \sqrt{2}\right|$, which we show in Figs. 3(c) and 3(d). The resonant driving of the Higgs mode by the terahertz pulse yields displacements of the indium ions of up to $3 \mathrm{pm}$, which corresponds to $1.5 \%$ of the interatomic distance. These displacements lie well below the Lindemann instability criterion that predicts melting of the crystal lattice when the displacements exceed $\sim 10 \%$ of the interatomic distance $[65,66]$. Surprisingly, the parametric excitation of the Goldstone mode yields indium ion displacements of up to $3 \mathrm{pm}$, comparable to the direct excitation of the Higgs mode, even for moderate linewidths. The induced displacements depend strongly on the linewidth of the Higgs mode $\kappa_{H}$ and show no dependence on the linewidth of the Goldstone mode $\kappa_{G}$. The maximal displacements of the Goldstone mode in Fig. 3(d) show the expected stochastic fluctuations due to the thermal noise. In contrast, Fig. 3(c) for the Higgs mode shows no fluctuations, because its amplitude reaches its maximum right after the arrival of the terahertz pulse and before the buildup of the Goldstone mode amplitude due to parametric downconversion, as seen in Fig. 3(a). The narrowest linewidths yield the largest amplitudes, despite having less overlap of components at the resonance condition, $Q_{G}=Q_{H} / 2$, suggesting that a major contribution to the overlap comes from the temporal envelope imposed on the Higgs mode due to the excitation by the terahertz pulse.

Conclusion.-Coherently excited IR- and Ramanactive phonons have been used to manipulate the properties of materials in recent years, most prominently leading to superconductivity far above the equilibrium critical temperature $[67,68]$, and light-induced ferroelectricity and ferroelectric switching [69-71]. The parametric excitation technique presented here adds silent phonons to the spectrum, closing the hitherto remaining gap in the range of coherently addressable phonon modes. The silent Goldstone mode in this work provides direct control over the phase of the primary order parameter in $\mathrm{InMnO}_{3}$ and we anticipate that the manipulation of other material properties connected to silent phonon modes will be explored in the future.

The coupling between the Higgs and Goldstone modes derives purely from symmetry considerations, and we expect that the dynamics described here are a general feature of the hexagonal manganites. Furthermore, the mechanism of parametric down-conversion requires only the existence of fully symmetric IR-active modes and therefore extends to all materials with broken inversion symmetry. The energy conversion efficiency of the mechanism outperforms ionic Raman scattering, in which only a fraction of the energy is transferred to other modes $[42,72-74]$, and is at par with predictions for the transfer of energy in the resonant phonon up-conversion process sum-frequency ionic Raman scattering [42,43,75]. The mechanism therefore circumvents the low efficiency of hyper-Raman scattering and makes silent phonons similarly accessible as IR- and Raman-active phonons. We suggest that techniques used in the field of ultrastrong lightmatter coupling [76] could be leveraged here by tuning the frequency of the IR-active mode into perfect resonance conditions with the second phonon mode, $Q_{\mathrm{IR}} / 2=Q_{2}$, in 
an optical cavity in order to increase the efficiency of the mechanism even further [77].

We point out that the parametric down-conversion described here is fundamentally different from the parametric amplification observed in a recent experiment [78], in which the temporal modulation of the frequency of the optical phonon mode results from a nonlinearity of the dielectric function in the phonon amplitude and no phononphonon coupling is involved. While it has been shown that parametric resonances of phonons may influence the superconducting critical temperature [79], the mechanism here is fundamentally different from the parametric amplification of light by an optically excited Higgs mode in superconductors discovered recently [21]. We further note that coherent energy transfer to a nearly silent phonon mode has been observed in a high-harmonic phonon upconversion process [44].

Finally, the generation of a large population of silent phonons may serve as a platform for higher-order nonlinear optical phenomena. Recent publications have indicated that driven Raman-active modes in solids or molecules may act as amplifiers [21] or transducers [80] of radiation through Raman processes down to the single-photon level. We suggest that similar mechanisms should be possible in higher order of the optical nonlinearity, using driven silent phonons that may amplify or transduce correlated pairs of photons through hyper-Raman processes.

We are grateful to Nicola Spaldin (ETH Zurich) and Hyeongrak Choi (MIT) for useful discussions and for feedback on the manuscript. This work was supported by the Swiss National Science Foundation under Project No. 184259, the DARPA DRINQS program under Award No. D18AC00014, the ERC Synergy project HERO under Grant Agreement No. 810451, as well as by Harvard University and ETH Zurich. This research was partially funded by the Gordon and Betty Moore Foundation under Grant No. GBMF8048 to P. N. Calculations were performed at the Swiss National Supercomputing Centre (CSCS) supported by Projects No. s889 and No. eth3 and at Harvard's Research Computing Facility.

*djuraschek@seas.harvard.edu prineha@seas.harvard.edu

[1] D. N. Basov, R. D. Averitt, and D. Hsieh, Towards properties on demand in quantum materials, Nat. Mater. 16, 1077 (2017).

[2] P. W. Higgs, Nobel lecture: Evading the Goldstone theorem, Rev. Mod. Phys. 86, 851 (2014).

[3] P. W. Anderson, Higgs, Anderson and all that, Nat. Phys. 11, 93 (2015).

[4] D. Podolsky, A. Auerbach, and D. P. Arovas, Visibility of the amplitude (Higgs) mode in condensed matter, Phys. Rev. B 84, 174522 (2011).

[5] L. Pollet and N. Prokof'ev, Higgs Mode in a TwoDimensional Superfluid, Phys. Rev. Lett. 109, 010401 (2012).
[6] M. Endres, T. Fukuhara, D. Pekker, M. Cheneau, P. Schauß, C. Gross, E. Demler, S. Kuhr, and I. Bloch, The 'Higgs' amplitude mode at the two-dimensional superfluid/Mott insulator transition, Nature (London) 487, 454 (2012).

[7] S. Hoinka, P. Dyke, M. G. Lingham, J. J. Kinnunen, G. M. Bruun, and C. J. Vale, Goldstone mode and pair-breaking excitations in atomic Fermi superfluids, Nat. Phys. 13, 943 (2017).

[8] A. Behrle, T. Harrison, J. Kombe, K. Gao, M. Link, J. S. Bernier, C. Kollath, and M. Köhl, Higgs mode in a strongly interacting fermionic superfluid, Nat. Phys. 14, 781 (2018).

[9] J. Léonard, A. Morales, P. Zupancic, T. Donner, and T. Esslinger, Monitoring and manipulating Higgs and Goldstone modes in a supersolid quantum gas, Science 358, 1415 (2017).

[10] C. M. Varma, Higgs Boson in superconductors, J. Low Temp. Phys. 126, 901 (2002).

[11] Y. Barlas and C. M. Varma, Amplitude or Higgs modes in $d$-wave superconductors, Phys. Rev. B 87, 054503 (2013).

[12] R. Matsunaga, Y. I. Hamada, K. Makise, Y. Uzawa, H. Terai, Z. Wang, and R. Shimano, Higgs Amplitude Mode in the BCS Superconductors $\mathrm{Nb}_{1-x} \mathrm{Ti}_{x} \mathrm{~N}$ Induced by Terahertz Pulse Excitation, Phys. Rev. Lett. 111, 057002 (2013).

[13] M. A. Méasson, Y. Gallais, M. Cazayous, B. Clair, P. Rodière, L. Cario, and A. Sacuto, Amplitude Higgs mode in the $2 \mathrm{H}-\mathrm{NbSe}_{2}$ superconductor, Phys. Rev. B 89, 060503 (R) (2014).

[14] R. Matsunaga, N. Tsuji, H. Fujita, A. Sugioka, K. Makise, Y. Uzawa, H. Terai, Z. Wang, H. Aoki, and R. Shimano, Light-induced collective pseudospin precession resonating with Higgs mode in a superconductor, Science 345, 1145 (2014).

[15] D. Sherman, U. S. Pracht, B. Gorshunov, S. Poran, J. Jesudasan, M. Chand, P. Raychaudhuri, M. Swanson, N. Trivedi, A. Auerbach, M. Scheffler, A. Frydman, and M. Dressel, The Higgs mode in disordered superconductors close to a quantum phase transition, Nat. Phys. 11, 188 (2015).

[16] A. F. Kemper, M. A. Sentef, B. Moritz, J. K. Freericks, and T. P. Devereaux, Direct observation of Higgs mode oscillations in the pump-probe photoemission spectra of electron-phonon mediated superconductors, Phys. Rev. B 92, 224517 (2015).

[17] D. Pekker and C. M. Varma, Amplitude/Higgs modes in condensed matter physics, Annu. Rev. Condens. Matter Phys. 6, 269 (2015).

[18] L. Schwarz, B. Fauseweh, N. Tsuji, N. Cheng, N. Bittner, H. Krull, M. Berciu, G. S. Uhrig, A. P. Schnyder, S. Kaiser, and D. Manske, Higgs spectroscopy of superconductors in nonequilibrium, Nat. Commun. 11, 287 (2020).

[19] H. Chu et al., New collective mode in superconducting cuprates uncovered by Higgs spectroscopy, arXiv:1901 .06675 .

[20] R. Shimano and N. Tsuji, Higgs mode in superconductors, Annu. Rev. Condens. Matter Phys. 11 (2020)..

[21] M. Buzzi, G. Jotzu, A. Cavalleri, J. I. Cirac, E. A. Demler, B. I. Halperin, M. D. Lukin, T. Shi, Y. Wang, and D. Podolsky, Higgs-mediated optical amplification in a nonequilibrium superconductor, arXiv:1908.10879v1. 
[22] H. Y. Liu, I. Gierz, J. C. Petersen, S. Kaiser, A. Simoncig, A. L. Cavalieri, C. Cacho, I. C. E. Turcu, E. Springate, F. Frassetto, L. Poletto, S. S. Dhesi, Z. A. Xu, T. Cuk, R. Merlin, and A. Cavalleri, Possible observation of parametrically amplified coherent phasons in $\mathrm{K}_{0.3} \mathrm{MoO}_{3}$ using time-resolved extreme-ultraviolet angle-resolved photoemission spectroscopy, Phys. Rev. B 88, 045104 (2013).

[23] R. Mankowsky, B. Liu, S. Rajasekaran, H. Y. Liu, D. Mou, X. J. Zhou, R. Merlin, M. Först, and A. Cavalleri, Dynamical Stability Limit for the Charge Density Wave in $\mathrm{K}_{0.3} \mathrm{MoO}_{3}$, Phys. Rev. Lett. 118, 116402 (2017).

[24] A. Zong, X. Shen, A. Kogar, L. Ye, C. Marks, D. Chowdhury, T. Rohwer, B. Freelon, S. Weathersby, R. Li, J. Yang, J. Checkelsky, X. Wang, and N. Gedik, Ultrafast manipulation of mirror domain walls in a charge density wave, Sci. Adv. 4, eaau5501 (2018).

[25] Ch. Rüegg, B. Normand, M. Matsumoto, A. Furrer, D. F. McMorrow, K. W. Krämer, H. U. Güdel, S. N. Gvasaliya, H. Mutka, and M. Boehm, Quantum Magnets under Pressure: Controlling Elementary Excitations in $\mathrm{TlCuCl}_{3}$, Phys. Rev. Lett. 100, 205701 (2008).

[26] A. Jain, M. Krautloher, J. Porras, G. H. Ryu, D. P. Chen, D. L. Abernathy, J. T. Park, A. Ivanov, J. Chaloupka, G. Khaliullin, B. Keimer, and B. J. Kim, Higgs mode and its decay in a two-dimensional antiferromagnet, Nat. Phys. 13, 633 (2017).

[27] S.-M. Souliou, J. Chaloupka, G. Khaliullin, G. Ryu, A. Jain, B. J. Kim, M. Le Tacon, and B. Keimer, Raman Scattering from Higgs Mode Oscillations in the Two-Dimensional Antiferromagnet $\mathrm{Ca}_{2} \mathrm{RuO}_{4}$, Phys. Rev. Lett. 119, 067201 (2017).

[28] D. Werdehausen, T. Takayama, M. Höppner, G. Albrecht, A. W. Rost, Y. Lu, D. Manske, H. Takagi, and S. Kaiser, Coherent order parameter oscillations in the ground state of the excitonic insulator $\mathrm{Ta}_{2} \mathrm{NiSe}_{5}$, Sci. Adv. 4, eaap8652 (2018).

[29] M. Vallone, Higgs and Goldstone modes in crystalline solids, Phys. Status Solidi B 257, 1900443 (2019).

[30] C. A. Kendziora, I. A. Sergienko, R. Jin, J. He, V. Keppens, B. C. Sales, and D. Mandrus, Goldstone-Mode Phonon Dynamics in the Pyrochlore $\mathrm{Cd}_{2} \mathrm{Re}_{2} \mathrm{O}_{7}$, Phys. Rev. Lett. 95, 125503 (2005).

[31] S. M. Nakhmanson and I. Naumov, Goldstone-like States in a Layered Perovskite with Frustrated Polarization: A FirstPrinciples Investigation of $\mathrm{PbSr}_{2} \mathrm{Ti}_{2} \mathrm{O}_{7}$, Phys. Rev. Lett. 104, 097601 (2010).

[32] J. Mangeri, K. C. Pitike, S. P. Alpay, and S. Nakhmanson, Amplitudon and phason modes of electrocaloric energy interconversion, npj Comput. Mater. 2, 16020 (2016).

[33] A. Marthinsen, S. M. Griffin, M. Moreau, T. Grande, T. Tybell, and S. M. Selbach, Goldstone-like phonon modes in a (111)-strained perovskite, Phys. Rev. Mater. 2, 014404 (2018).

[34] Q. N. Meier, A. Stucky, J. Teyssier, S. M. Griffin, D. van der Marel, and N. A. Spaldin, Manifestion of structural Higgs and Goldstone modes in the hexagonal manganites, arXiv: 1912.04942.

[35] N. N. Kovaleva, O. E. Kusmartseva, K. I. Kugel, A. A. Maksimov, D. Nuzhnyy, A. M. Balbashov, E. I. Demikhov,
A. Dejneka, V. A. Trepakov, F. V. Kusmartsev, and A. M. Stoneham, Anomalous multi-order Raman scattering in $\mathrm{LaMnO}_{3}$ : A signature of quantum lattice effects in a Jahn-Teller crystal, J. Phys Condens. Matter 25, 155602 (2013).

[36] S. De Silvestri, J. G. Fujimoto, E. P. Ippen, E. B. Gamble, Jr., L. R. Williams, and K. A. Nelson, Femtosecond timeresolved measurements of optic phonon dephasing by impulsive stimulated Raman scattering in $\alpha$-perylene crystal from 20 to $300 \mathrm{~K}$, Chem. Phys. Lett. 116, 146 (1985).

[37] R. Merlin, Generating coherent $\mathrm{THz}$ phonons with light pulses, Solid State Commun. 102, 207 (1997).

[38] M. Först, C. Manzoni, S. Kaiser, Y. Tomioka, Y. Tokura, R. Merlin, and A. Cavalleri, Nonlinear phononics as an ultrafast route to lattice control, Nat. Phys. 7, 854 (2011).

[39] A. Subedi, A. Cavalleri, and A. Georges, Theory of nonlinear phononics for coherent light control of solids, Phys. Rev. B 89, 220301(R) (2014).

[40] D. Nicoletti and A. Cavalleri, Nonlinear light-matter interaction at terahertz frequencies, Adv. Opt. Photonics 8, 401 (2016).

[41] S. Maehrlein, A. Paarmann, M. Wolf, and T. Kampfrath, Terahertz Sum-Frequency Excitation of a Raman-Active Phonon, Phys. Rev. Lett. 119, 127402 (2017).

[42] D. M. Juraschek and S. F. Maehrlein, Sum-frequency ionic Raman scattering, Phys. Rev. B 97, 174302 (2018).

[43] A. A. Melnikov, K. N. Boldyrev, Yu. G. Selivanov, V. P. Martovitskii, S. V. Chekalin, and E. A. Ryabov, Coherent phonons in a $\mathrm{Bi}_{2} \mathrm{Se}_{3}$ film generated by an intense singlecycle THz pulse, Phys. Rev. B 97, 214304 (2018).

[44] M. Kozina, M. Fechner, P. Marsik, T. van Driel, J. M. Glownia, C. Bernhard, M. Radovic, D. Zhu, S. Bonetti, U. Staub, and M. C. Hoffmann, Terahertz-driven phonon upconversion in $\mathrm{SrTiO}_{3}$, Nat. Phys. 15, 387 (2019).

[45] C. L. Johnson, B. E. Knighton, and J. A. Johnson, Distinguishing Nonlinear Terahertz Excitation Pathways with Two-Dimensional Spectroscopy, Phys. Rev. Lett. 122, 073901 (2019).

[46] B. E. Knighton, R. T. Hardy, C. L. Johnson, L. M. Rawlings, J. T. Woolley, C. Calderon, A. Urrea, and J. A. Johnson, Terahertz waveform considerations for nonlinearly driving lattice vibrations, J. Appl. Phys. 125, 144101 (2019).

[47] S. W. Teitelbaum, T. Henighan, Y. Huang, H. Liu, M. P. Jiang, D. Zhu, M. Chollet, T. Sato, É. D. Murray, S. Fahy, S. O'Mahony, T. P. Bailey, C. Uher, M. Trigo, and D. A. Reis, Direct Measurement of Anharmonic Decay Channels of a Coherent Phonon, Phys. Rev. Lett. 121, 125901 (2018).

[48] S. M. Griffin, M. Lilienblum, K. T. Delaney, Y. Kumagai, M. Fiebig, and N. A. Spaldin, Scaling Behavior and Beyond Equilibrium in the Hexagonal Manganites, Phys. Rev. X 2, 041022 (2012).

[49] S. Artyukhin, K. T. Delaney, N. A. Spaldin, and M. Mostovoy, Landau theory of topological defects in multiferroic hexagonal manganites, Nat. Mater. 13, 42 (2014).

[50] S. H. Skjærvø, Q. N. Meier, M. Feygenson, N. A. Spaldin, S. J. L. Billinge, E. S. Bozin, and S. M. Selbach, Unconventional Continuous Structural Disorder at the Order-Disorder Phase Transition in the Hexagonal Manganites, Phys. Rev. X 9, 031001 (2019). 
[51] Y. Kumagai, A. A. Belik, M. Lilienblum, N. Leo, M. Fiebig, and N. A. Spaldin, Observation of persistent centrosymmetricity in the hexagonal manganite family, Phys. Rev. B 85, 174422 (2012).

[52] F.-T. Huang, X. Wang, S. M. Griffin, Y. Kumagai, O. Gindele, M.-W. Chu, Y. Horibe, N. A. Spaldin, and S.-W. Cheong, Duality of Topological Defects in Hexagonal Manganites, Phys. Rev. Lett. 113, 267602 (2014).

[53] X. Gonze et al., Recent developments in the ABINIT software package, Comput. Phys. Commun. 205, 106 (2016).

[54] B. Amadon, F. Jollet, and M. Torrent, $\gamma$ and $\beta$ cerium: LDA + U ground-state parameters, Phys. Rev. B 77, 155104 (2008).

[55] M. Torrent, F. Jollet, F. Bottin, G. Zérah, and X. Gonze, Implementation of the projector augmented-wave method in the ABINIT code: Application to the study of iron under pressure, Comput. Mater. Sci. 42, 337 (2008).

[56] J. J. Mortensen, L. B. Hansen, and K. W. Jacobsen, Realspace grid implementation of the projector augmented wave method, Phys. Rev. B 71, 035109 (2005).

[57] J. E. Medvedeva, V. I. Anisimov, M. A. Korotin, O. N. Mryasov, and A. J. Freeman, The effect of Coulomb correlation and magnetic ordering on the electronic structure of two hexagonal phases of ferroelectromagnetic $\mathrm{YMnO}_{3}$, J. Phys. Condens. Matter 12, 4947 (2000).

[58] X. Gonze and C. Lee, Dynamical matrices, Born effective charges, dielectric permittivity tensors, and interatomic force constants from density-functional perturbation theory, Phys. Rev. B 55, 10355 (1997).

[59] Xavier Gonze, First-principles responses of solids to atomic displacements and homogeneous electric fields: Implementation of a conjugate-gradient algorithm, Phys. Rev. B 55, 10337 (1997).

[60] B. Liu, H. Bromberger, A. Cartella, T. Gebert, M. Först, and A. Cavalleri, Generation of narrowband, high-intensity, carrier-envelope phase-stable pulses tunable between 4 and 18 THz, Opt. Lett. 42, 129 (2017).

[61] A. Togo, L. Chaput, and I. Tanaka, Distributions of phonon lifetimes in Brillouin zones, Phys. Rev. B 91, 094306 (2015).

[62] M. N. Iliev, H.-G. Lee, V. N. Popov, M. V. Abrashev, A. Hamed, R. L. Meng, and C. W. Chu, Raman- and infraredactive phonons in hexagonal $\mathrm{YMnO}_{3}$ : Experiment and lattice-dynamical calculations, Phys. Rev. B 56, 2488 (1997).

[63] S. Joubaud, N. B. Garnier, and S. Ciliberto, Fluctuation theorems for harmonic oscillators, J. Stat. Mech. (2007) P09018.

[64] M. Gitterman, The Noisy Oscillator (World Scientific, Singapore, 2013).

[65] F. A. Lindemann, The calculation of molecular Eigenfrequencies, Phys. Z. 11, 609 (1910), https://ntrs.nasa .gov/search.jsp?R=19840027015.
[66] K. Sokolowski-Tinten, C. Blome, J. Blums, A. Cavalleri, C. Dietrich, A. Tarasevitch, I. Uschmann, E. Förster, M. Kammler, M. Horn-von Hoegen, and D. von der Linde, Femtosecond X-ray measurement of coherent lattice vibrations near the Lindemann stability limit, Nature (London) 422, 287 (2003).

[67] R. Mankowsky, A. Subedi, M. Först, S. O. Mariager, M. Chollet, H. T. Lemke, J. S. Robinson, J. M. Glownia, M. P. Minitti, A. Frano, M. Fechner, N. A. Spaldin, T. Loew, B. Keimer, A. Georges, and A. Cavalleri, Nonlinear lattice dynamics as a basis for enhanced superconductivity in $\mathrm{YBa}_{2} \mathrm{Cu}_{3} \mathrm{O}_{6.5}$, Nature (London) 516, 71 (2014).

[68] M. Mitrano, A. Cantaluppi, D. Nicoletti, S. Kaiser, A. Perucchi, S. Lupi, P. Di Pietro, D. Pontiroli, M. Riccò, S. R. Clark, D. Jaksch, and A. Cavalleri, Possible light-induced superconductivity in $\mathrm{K}_{3} \mathrm{C}_{60}$ at high temperature, Nature (London) 530, 461 (2016).

[69] R. Mankowsky, A. von Hoegen, M. Först, and A. Cavalleri, Ultrafast Reversal of the Ferroelectric Polarization, Phys. Rev. Lett. 118, 197601 (2017).

[70] T. Nova, A. Disa, M. Fechner, and A. Cavalleri, Metastable ferroelectricity in optically strained $\mathrm{SrTiO}_{3}$, Science 364, 1075 (2019).

[71] X. Li, T. Qiu, J. Zhang, E. Baldini, J. Lu, A. M. Rappe, and K. A. Nelson, Terahertz field-induced ferroelectricity in quantum paraelectric $\mathrm{SrTiO}_{3}$, Science 364, 1079 (2019).

[72] M. Fechner and N. A. Spaldin, Effects of intense optical phonon pumping on the structure and electronic properties of yttrium barium copper oxide, Phys. Rev. B 94, 134307 (2016).

[73] D. M. Juraschek, M. Fechner, and N. A. Spaldin, Ultrafast Structure Switching through Nonlinear Phononics, Phys. Rev. Lett. 118, 054101 (2017).

[74] M. Gu and J. M. Rondinelli, Nonlinear phononic control and emergent magnetism in Mott insulating titanates, Phys. Rev. B 98, 024102 (2018).

[75] D. M. Juraschek and N. A. Spaldin, Orbital magnetic moments of phonons, Phys. Rev. Mater. 3, 064405 (2019).

[76] J. Flick, N. Rivera, and P. Narang, Strong light-matter coupling in quantum chemistry and quantum photonics, Nanophotonics 7, 1479 (2018).

[77] D. M. Juraschek, T. Neuman, J. Flick, and P. Narang, Cavity control of nonlinear phononics, arXiv:1912.00122.

[78] A. Cartella, T. F. Nova, M. Fechner, R. Merlin, and A. Cavalleri, Parametric amplification of optical phonons, Proc. Natl. Acad. Sci. U.S.A. 115, 12148 (2018).

[79] M. Knap, M. Babadi, G. Refael, I. Martin, and E. Demler, Dynamical Cooper pairing in nonequilibrium electronphonon systems, Phys. Rev. B 94, 214504 (2016).

[80] P. Roelli, D.-M. Cano, T. J. Kippenberg, and C. Galland, Molecular platform for frequency upconversion at the single-photon level, arXiv:1910.11395. 